\title{
Shortwave broadband Transmission System with Fountain Code Technology based on MFC
}

\author{
Jiang Bian ${ }^{1, a}$, XinLe $\mathrm{Yu}^{2, \mathrm{~b}}$ and Hao Duan ${ }^{2, \mathrm{c}}$ \\ ${ }^{1}$ School of Computing, Communication University of China, Beijing 100024, China; \\ ${ }^{2}$ School of Computing,Communication University of China, Beijing 100024, China.

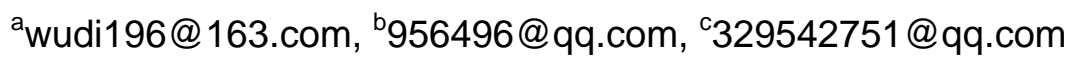

Keywords: shortwave broadband, MFC, Fountain code

Abstract. This paper briefly introduced a short-wave transmission system with fountain code technology based on MFC.This system can realize the real-time transmission of multi-media files.

\section{Introduction}

In recent years,with the advent of science and technology of communication industry,short-wave communication has been one of the major forms in wireless communication.Frequency between $3 \mathrm{MHz}$ and30MHz. Wave length between $100 \mathrm{~m}$ and $10 \mathrm{~m}$, which mostly use ionized stratum to realize data transmission.It can realize the long distance communication without relay station.Transmission distance is very far which around the earth,It is also a major means of telecommunication.In general,the equipment of short-wave hascharacteristics including smaller size, lower cost and easy maintenance,which can achieve long distance communication by low transmitted power.

After the analog communication and digital communication appear,the third wireless communication technology was born----software radio technology.Now,the transmission and using of information play important role in various industries. The market needs, an innovative technology that can greatly improve the ability of informationcommunicate. When software radio technology was put forward,it became the research focus in the field of the research,and it soon became a main method of telecommunication.

In this paper,we implement the system of short-wave file transmission in Windows platform based on MFC software,with fountain codes to achieve the system of multi-media file transmission.The remainder of this paper is organized as follows:Section II describes the FountainCode Technology. In Section III the design of software will be performed. In Section IV,We present our conclusions.

\section{FountainCodeTechnology}

The concept of digital fountain was put forward by John Byers and Michael Luby in 1998 and it was born on the basis of the Forward Error Correction (FEC) technology [1] [2]. The main point of digital fountain is similar to collecting water with glass under fountain and we just need to consider if glassis full of water. Similarly, the receiver can decode successfully only when it receive a sufficient number of encoded symbols and we don' $t$ need to know the order and resource of these symbols.

Digital fountain is only a concept in broadcast application when it was putted forward. The concept was achieved in implementation when M.Luby proposed LT(Luby Transform)codes in 2002 [3]. Coding principle of LT codes as shown in Fig.1. $s_{1}, s_{2}, s_{2}, \cdots, s_{k}$ are source characters, and $O_{1}, O_{2}, O_{3}, \cdots \cdots$ are output characters which has been encoded. $O_{1}=S_{1}+S_{3}, O_{2}=S_{2}+S_{2}, O_{a}=S_{1}+$ $s_{K} \cdots \cdots$ and the basic steps of coding are as follows:

1. Divide the original data for K source symbols of the same length and fill 0 in the last symbol if its length is insufficient; 
2. Get degree $d_{\mathrm{i}}$ according to the degree distribution $\rho(\mathrm{d})$; and $d_{\mathrm{i}}$ is the number of source symbols that to generate a output symbol ${ }_{k}$;

3. Select $d_{i}$ distinct input symbols as neighbors of the encoding symbol.
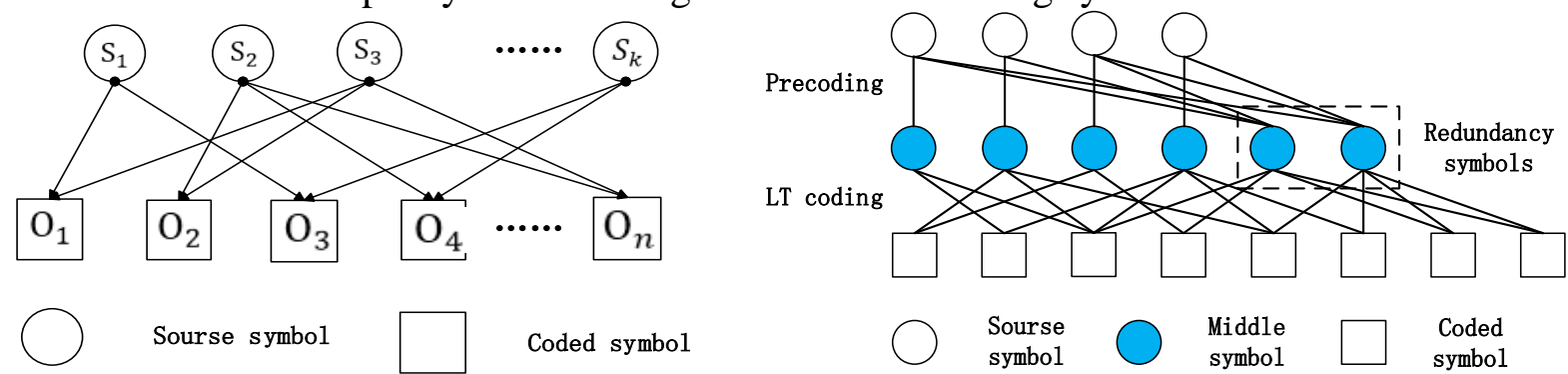

Fig1. Principle of LT codingFig 2. Principle of Raptor coding

The value of the encoding symbols is the exclusive-or of the $d_{i}$ neighbors. However, if a source character without generating any output characters, namely the encoded symbols not fully cover the original data, then the receiver unable to restore all the file and resulting in decoding failure. To solve this problem, LT coding need to generate some encoded characters has a large degree of value. Therefore it will consume a large amount of complicated XOR operation.

To overcome the shortcomings, Shokrollahi proposed Raptor code which was an extension of LT-codes. The principle of Raptor coding as shown in Fig.2, and it mainly composed of two processes which is precodingand LT coding. Firstly, given k source symbols and the pre-code first encodes these symbols into a code word of length $\mathrm{n}$. Secondly, conduct the LT encoding process by the middle characters.Because of using the error correction capability of traditional error correcting code, the Raptor code does not need to restore all the intermediate source symbols when decoding, all of the source symbols can be obtained and thus improve the success rate of decoding.

In recent years, the fountain code technology received extensive attention of the academia. Due to its huge technical advantages, fountain codes have been applied to many places, including deep space communication, multimedia broadcast and multicast, wireless transmission, mass data storage and other fields. But what we should realize is that we still need to improve it try our best.

\section{Software Design}

MFC (Microsoft Foundation Class) is one of Application Framework.MFC is not just a simple interface development system.Althoughmost of the MFC class used for interface development associated with a window, there are a lot of classes are not associated with a window, the role of the class is not an interface and it don't realize the control of a window object, such as creation and destroying.Some MFC in the WinDOS (for the most part written in MFC program in WinDOS running) implemented class in internal processing, such as database management, etc.

This system is divided into two parts of transmitter and receiver, and the corresponding signal processing flow diagram is shown in fig 3

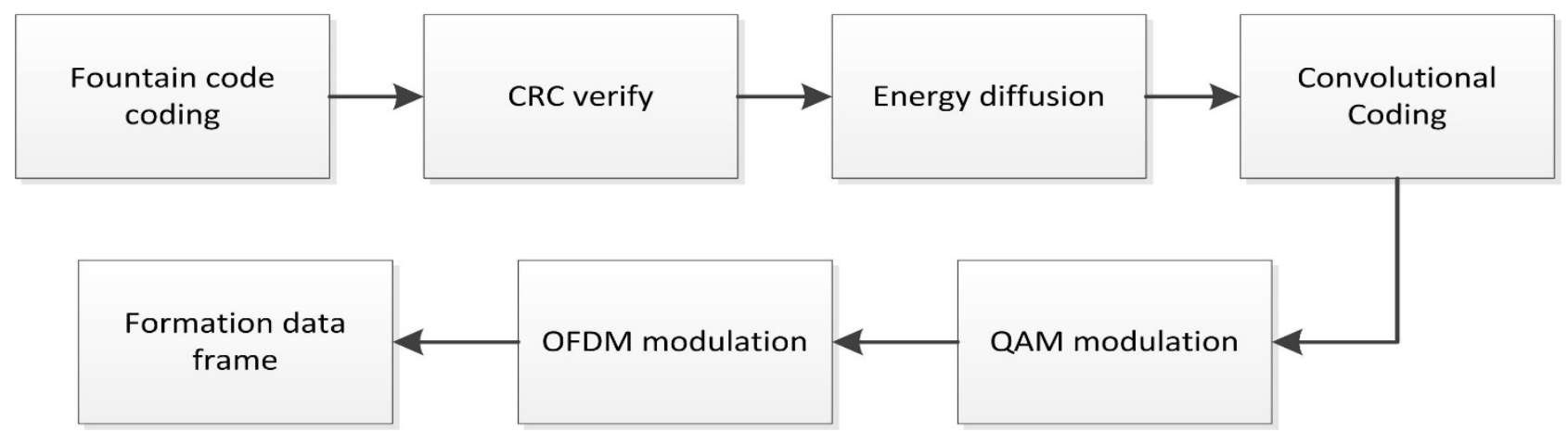

Fig 3. Signal Processing Flow Chart of Transmitting Terminal

The software operating platform of this system are implemented on Windows platform. Code is written by $\mathrm{C} / \mathrm{C}++$. The whole software system consists of a transmitter and a receiver. In Fig.4 the integrated short-wave system is shown.The transmitter interface as shown in Fig.5. 


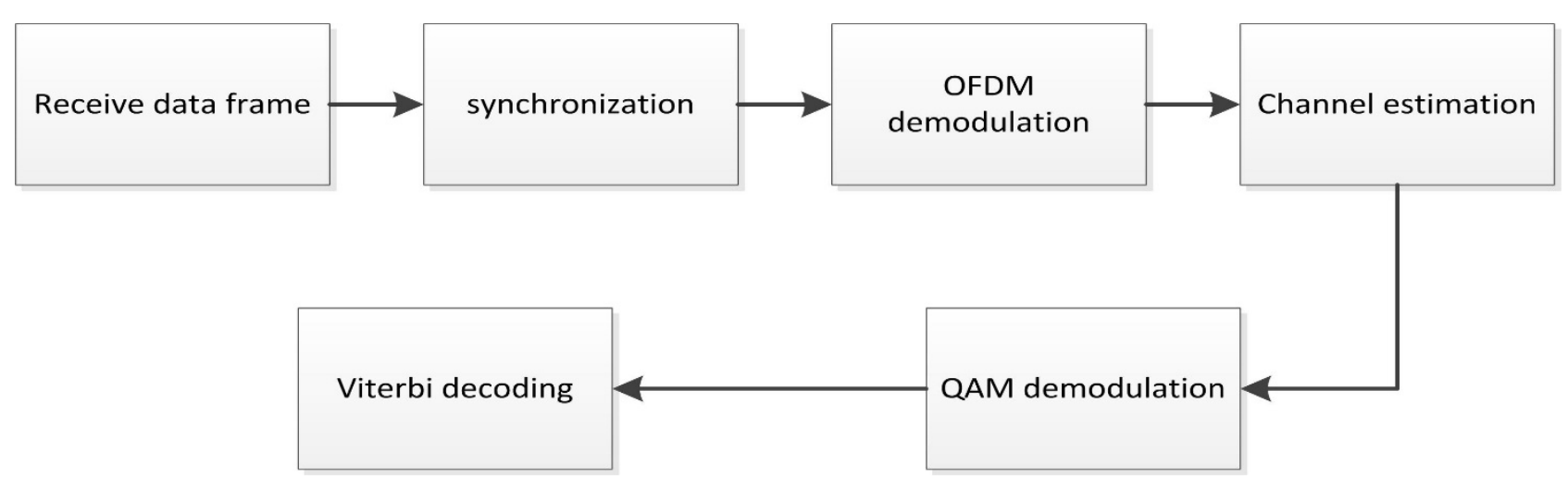

Fig 4. Signal Processing Flow Chart of Receiving Terminal

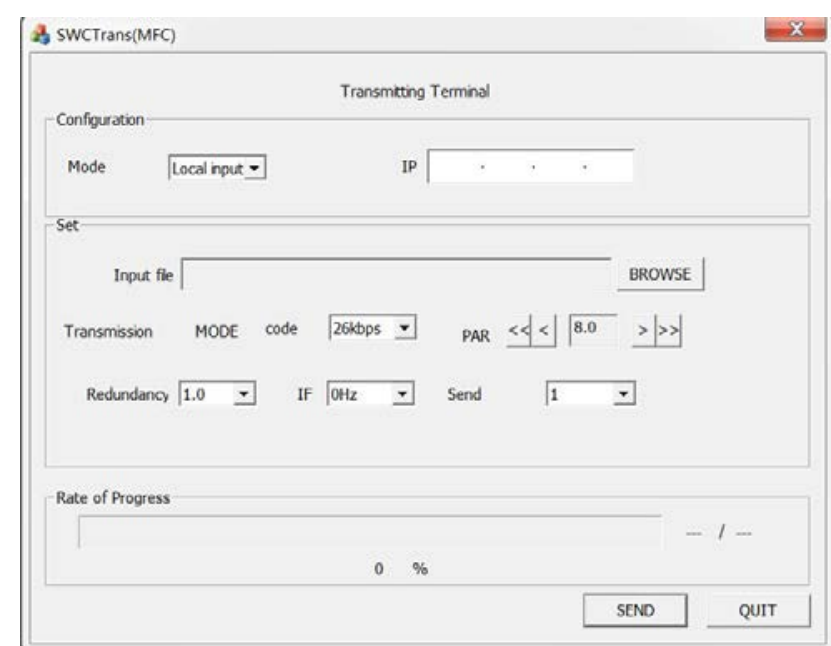

Fig 5.Transmitting Terminal UI

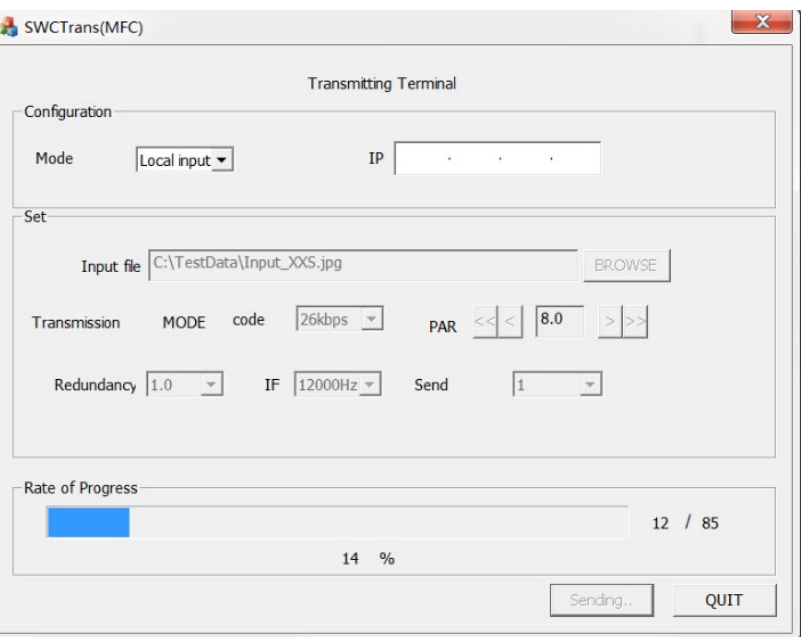

Fig 6.Transmitting in standard-line Mode

The main function of transmitter is selecting the path of the file (audio or data files) and transmitter signal to the corresponding receiver in shortwave communication way.Main parameters include transfer mode, the choice of modulation mode, coding, etc.The main parameters is introduced as list:

1. Redundancy: The data code may mutations in data transmission due to attenuation or interference.For this case,we must increase the length of several binary code,make corresponding data redundancy,to improve the anti-interference ability of data code.There are four redundancy parameter in this system,0.1,0.3,0.5 and 1.0.

2.The code rate: code rate base on QAM modulation choose and encoding, as same as quadrature amplitude modulation (am), which amplitude and phase change at the same time, belongs to the constant envelope modulation in $2 \mathrm{~d}$ QAM is orthogonal carrier modulation technology and the combination of multilevel amplitude shift keying.There are three different logical channel corresponding to different ways of QAM modulation in the channel coding.In this system, we have three chooses depend on factors such as transfer mode and channel coding rate is different.

1) 4 QAM - can provide a more robust error performance

2) 16 QAM - can provide higher spectrum efficiency

3) 64 QAM - can provide higher spectrum efficiency.

And specific choose QAM modulation method is selected according to the channel andtransmission mode. 


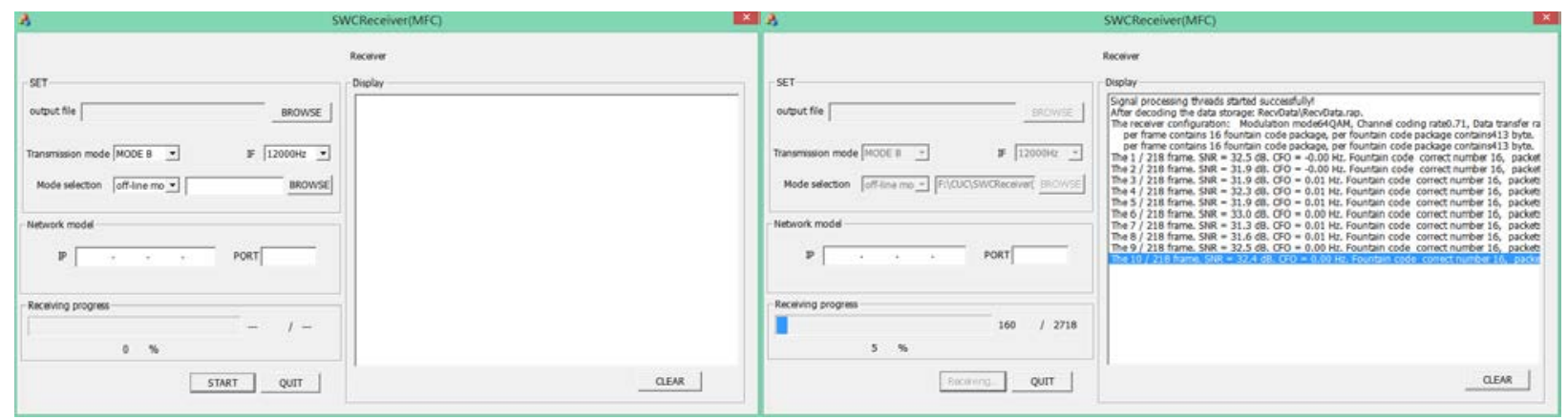

Fig 7. Receiving Terminal UI

Fig 8. Receiving Terminal Receive Data

Data selection parameters at the receiving terminal include the output file path,transfer mode, intermediate frequency, mode selection, etc.Also including the function of screen display, the progress of system can be displayed on the screen, feedback to staff.Mode selection includes two modes: standard mode and offline mode.Standard model is the transmitting terminal to send data, at the same time, the receiving terminal receives the data, and will decode the received data, restore to the original data.

Both of two parameters are important that transmission mode and intermediate frequency of transmitting terminal and receiving terminal must be same, otherwise unable to properly receive.

In the transmitter end to choose the file to be sent, parameter selection is completed, click send (testing, data on the PC from the sound card output).Send the progress at the transmitter end interface visual display.

At this point, the receiving terminal read data from the sound card,decode data frames, get the original data, complete data transmission.Besides,it will realize transmit data by network transmission.On figure 9,this picture shows when user choose input by internet access,and input IP,this system can receive data from internet by socket.Next,data will be transmit to receiving terminal.This process as same as standard mode.Receiving terminal choose parameter same as transmitting terminal,it will receiving data if check start button before transmitting terminal had send data over.The IP and PORT on receiving terminal means system will feedback information to the software that can show the data information.Figure 9 to 12 will show this process.

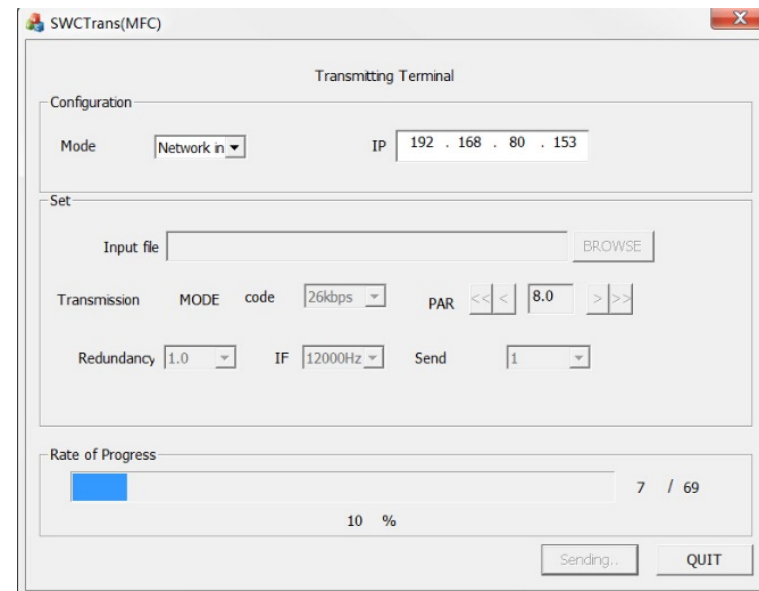

Fig 9. Transmitting Terminal UI

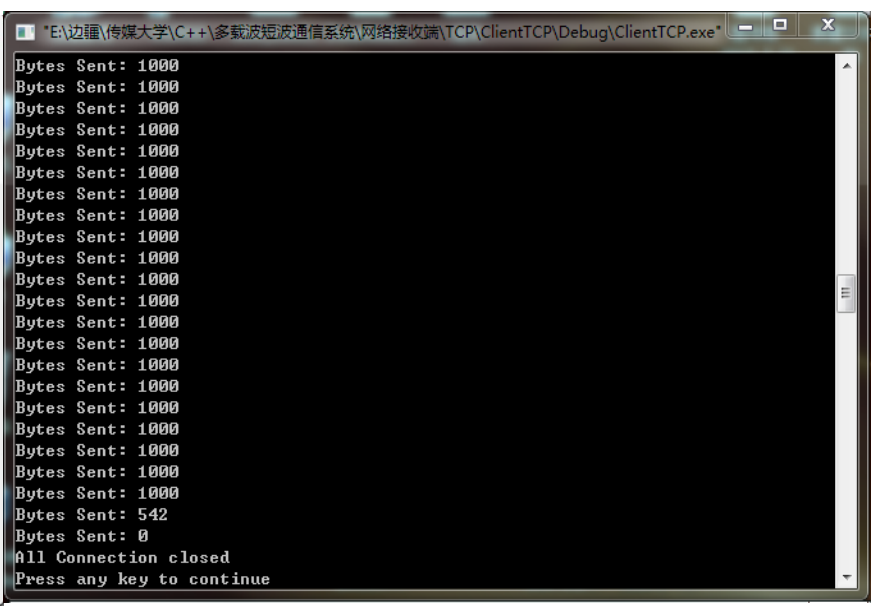

Fig 10. Client UI 


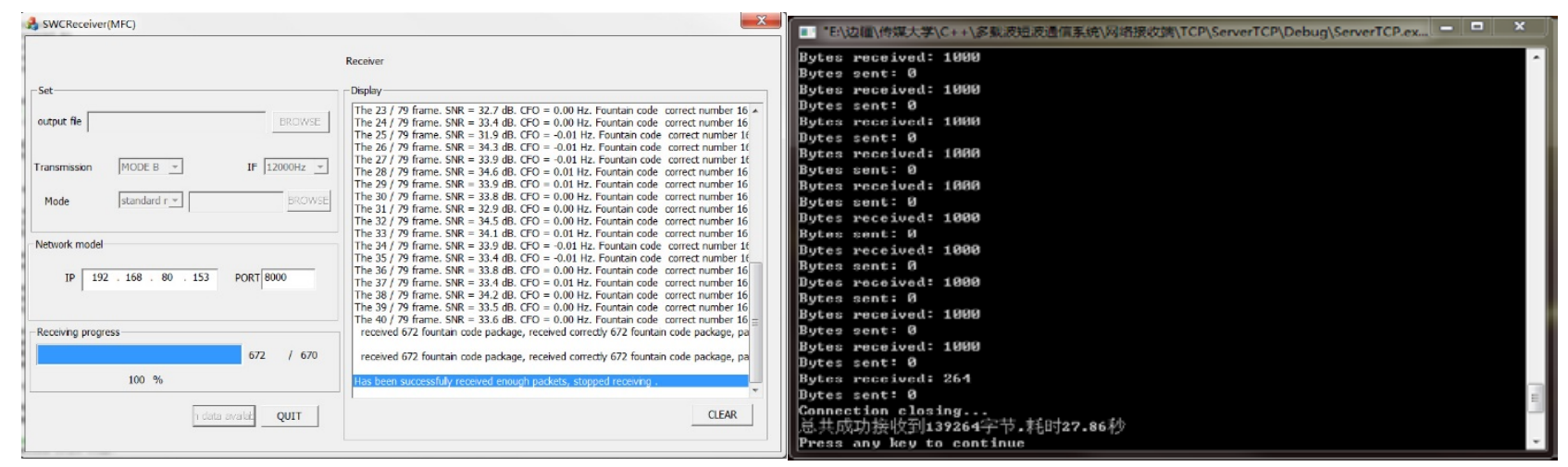

Fig 11. Data Received SucceedFig 12. Server UI

\section{Summary}

As an important erasure codes, digital fountain code with spending small decoding , approaching channel capacity and effective erasure performance characteristics, in the transmission reliability and operation effectiveness, digital fountain codes have greater advantage, have a good application prospect in the file transfer.The work of this paper include:

(1) We introduced the digital fountain code in brief, and analyzed the first practical application of fountain codes, the basic coding principle of LT codes, and Raptor code coding principle which based on LT fountain codes. This paper introduces the performance advantages of digital fountain codes and application scenarios wide.

(2)We tested software performance in the real environment, compared with the traditional way of data carousel, application of fountain codes technology will improve the transmission efficiency, especially in the case of transmit large file, it obvious advantages. brief introduce the characteristics of short-wave communication, summarized the process of the system.

We limited ability, existing problems in this system, need continuous improvement in practical application,to make better.Such as

1) optimize the fountain code algorithm

2) adding features, such as automatic send several times, path selection, etc.

\section{Acknowledgment}

This work is supported by a grant from National Key Technology R\&D Program of China under contract No. 2014BAK10B01.

\section{References}

[1]. JunjieHou. Dissecting MFC [M].Huazhong university of science and technology press.2001.p87-165

[2]. WeitongHuang ,RuixiaYao. Visual $\mathrm{c}++$ programming tutorial [M]. China Machine press.p61-72

[3]. Yong Yang, Bing Liu, Lin Zhang. Visual C++ 2010 program design case tutorial [M]. China Machine press.2012.p90-107

[4]. Dong Li. Digital Multimedia[M]. Electronic Industry Press.2001.p145-148

[5]. Xin Sun. VC + + depth explanation [M]. Electronic Industry Press.2006.p78-81 